\title{
THE
}

\section{On the stability of solutions of certain systems of differential equations with piecewise constant argument}

\author{
S. R. Grace \\ Mustafa Kulenović \\ University of Rhode Island, mkulenovic@uri.edu \\ H. El-Metwally \\ University of Rhode Island
}

Follow this and additional works at: https://digitalcommons.uri.edu/math_facpubs

Terms of Use

All rights reserved under copyright.

\section{Citation/Publisher Attribution}

Grace, S. R., Kulenović, M. R. S., \& El-Metwally, H. Czechoslovak Mathematical Journal (2002) 52:449. http://doi.org/10.1023/A:1021707225207

Available at: http://doi.org/10.1023/A:1021707225207

This Article is brought to you for free and open access by the Mathematics at DigitalCommons@URI. It has been accepted for inclusion in Mathematics Faculty Publications by an authorized administrator of DigitalCommons@URI.For more information, please contact digitalcommons-group@uri.edu. 


\section{ON THE STABILITY OF SOLUTIONS OF CERTAIN SYSTEMS \\ OF DIFFERENTIAL EQUATIONS WITH PIECEWISE CONSTANT ARGUMENT}

S. R. Grace, Orman, M. R. S. Kulenović, and H. El-Metwally*, Kingston

(Received March 22, 1999)

Abstract. We obtain some sufficient conditions for the existence of the solutions and the asymptotic behavior of both linear and nonlinear system of differential equations with continuous coefficients and piecewise constant argument.

Keywords: asymptotic behavior, delay, stability

MSC 2000: 34K20, 34K25, 39A10

\section{INTRODUCTION}

In this paper we study the nonlinear differential equation

$$
x^{\prime}(t)+A(t) x(t)=B(t) x\left(\left[t+\frac{1}{2}\right]\right)+f\left(t, x(t), x\left(\left[t+\frac{1}{2}\right]\right)\right)
$$

where $A(t)$ and $B(t)$ are $r \times r$ continuous matrices, $f: \mathbb{R} \times \mathbb{R}^{r} \times \mathbb{R}^{r} \rightarrow \mathbb{R}^{r}$ is a continuous function on $\mathbb{R} \times \mathbb{R}^{2 r}, x$ is a $r$-vector, and [.] is the integer part of a number.

Also we will investigate the equation

$$
x^{\prime}(t)+A(t) x(t)=B(t) x\left(\left[t+\frac{1}{2}\right]\right)
$$

with continuous matrices $A$ and $B$.

* On leave from Department of Mathematics, Faculty of Science, University of Mansoura, Mansoura, Egypt. 
Differential equations with piecewise constant argument (EPCA) represent a hybrid of continuous and discrete dynamical systems and therefore combine the properties of both the differential and difference equations. These equations have many applications in the control theory and certain biomedical models [3].

The research of first order differential equations with piecewise constant arguments of delay and advanced type was initiated by Cooke and Wiener [4] and Shah and Wiener [7]. A lot of results concerning the scalar versions of equation (1) have been studied in $[1,5,8]$ and the references cited therein.

Our goal in this article is to derive the sufficient conditions for the existence of solutions of equations (1) and (2) and the global asymptotic stability of equations (1) and (2). An important tool in achieving this goal will be the variant of the variation of constant formula for equation (2).

We note that the results related to Theorems 1 and 2 of this paper for equations of the form (2) with constant matrices $A$ and $B$ are included in Theorem 1 [9]. Also results concerning the existence and uniqueness are extensions of Theorem 1.1 [2].

\section{Main Results}

By a solution of equation $(1)$ on $(-\infty, \infty)$ we mean a function $x(t)$ satisfying the conditions

(i) $x(t)$ is continuous on $(-\infty, \infty)$;

(ii) the derivative $x^{\prime}(t)$ exists everywhere, with the possible exception of the halfinteger points $n+\frac{1}{2}$, where one-sided derivatives exist;

(iii) Eq. (1) is satisfied on each interval $\left[n-\frac{1}{2}, n+\frac{1}{2}\right)$, with integer $n$.

In what follows we will use the norm of a $p \times q$ matrix $A$ as

$$
|A|=\max \left\{\left|a_{i j}\right|: i=1,2, \ldots, p \text { and } j=1,2, \ldots, q \text { for } p \leqslant q\right\} .
$$

The trivial solution of equation (1) is said to be globally asymptotically stable if every solution $x(t)$ of equation (1) tends to zero as $t \rightarrow \infty$.

I.

In this part, we prove our main results for equation (2). In Theorem 1 and Theorem 2 below we will establish sufficient conditions for the existence and uniqueness of the solutions of Eq. (2). These results follow from the representation of the solutions of Eq. (2).

Theorem 2.1. Let the matrix

$$
C_{n}=I-\int_{n+\frac{1}{2}}^{n+1} B(u) \exp \left\{-\int_{u}^{n+1} A(s) \mathrm{d} s\right\} \mathrm{d} u, \quad n=0,1, \ldots
$$


be non-singular, then Eq. (2) has a unique solution on $[0, \infty)$ given by

$$
x(t)=\left[F(t, n)+\int_{n}^{t} B(s) F(t, s) \mathrm{d} s\right] a_{n},
$$

where $a_{n}$ is a solution of the difference equation

$$
a_{n+1}=C_{n}^{-1} D_{n} a_{n}, \quad n=0,1, \ldots,
$$

$C_{n}$ is defined above,

$$
D_{n}=F(n+1, n)+\int_{n}^{n+\frac{1}{2}} B(s) F(n+1, s) \mathrm{d} s
$$

and

$$
F(u, v)=\exp \left\{-\int_{v}^{u} A(s) \mathrm{d} s\right\}
$$

Proof. For $t \in\left[n-\frac{1}{2}, n+\frac{1}{2}\right)$, Eq. (2) reduces to

$$
x^{\prime}(t)+A(t) x(t)=B(t) a_{n}, \quad a_{n}=x(n) .
$$

Integrating Eq. (5) from $n$ to $t<n+\frac{1}{2}$ gives

$$
x(t)-\exp \left\{-\int_{n}^{t} A(s) \mathrm{d} s\right\} a_{n}=\exp \left\{-\int_{n}^{t} A(s) \mathrm{d} s\right\} \int_{n}^{t} B(u) \exp \left\{\int_{n}^{u} A(s) \mathrm{d} s\right\} \mathrm{d} u a_{n} .
$$

Hence we obtain

$$
x(t)=\left[\exp \left\{-\int_{n}^{t} A(s) \mathrm{d} s\right\}+\int_{n}^{t} B(u) \exp \left\{-\int_{u}^{t} A(s) \mathrm{d} s\right\} \mathrm{d} u\right] a_{n},
$$

which is equivalent to

$$
x(t)=\left[F(t, n)+\int_{n}^{t} B(u) F(t, u) \mathrm{d} u\right] a_{n},
$$

where

$$
F(u, v)=\exp \left\{-\int_{v}^{u} A(s) \mathrm{d} s\right\}
$$

Letting $t \rightarrow n+\frac{1}{2}$, we have

(6) $x\left(n+\frac{1}{2}\right)=\left[\exp \left\{-\int_{n}^{n+\frac{1}{2}} A(s) \mathrm{d} s\right\}+\int_{n}^{n+\frac{1}{2}} B(u) \exp \left\{-\int_{u}^{n+\frac{1}{2}} A(s) \mathrm{d} s\right\} \mathrm{d} u\right] a_{n}$. 
Again, for $t \in\left[n+\frac{1}{2}, n+\frac{3}{2}\right)$, integrating Eq. (2) from $n+1$ to $t$ we obtain

$$
x(t)=\left[\exp \left\{-\int_{n+1}^{t} A(s) \mathrm{d} s\right\}+\int_{n+1}^{t} B(u) \exp \left\{-\int_{u}^{t} A(s) \mathrm{d} s\right\} \mathrm{d} u\right] a_{n+1} .
$$

Then as $t \rightarrow n+\frac{1}{2}$, the continuity of $x(t)$ yields

$$
x\left(n+\frac{1}{2}\right)=\left[\exp \left\{-\int_{n+1}^{n+\frac{1}{2}} A(s) \mathrm{d} s\right\}+\int_{n+1}^{n+\frac{1}{2}} B(u) \exp \left\{-\int_{u}^{n+\frac{1}{2}} A(s) \mathrm{d} s\right\} \mathrm{d} u\right] a_{n+1} .
$$

Equations (6) and (7) imply

$$
\begin{aligned}
& {\left[\exp \left\{-\int_{n+1}^{n+\frac{1}{2}} A(s) \mathrm{d} s\right\}+\int_{n+1}^{n+\frac{1}{2}} B(u) \exp \left\{-\int_{u}^{n+\frac{1}{2}} A(s) \mathrm{d} s\right\} \mathrm{d} u\right] a_{n+1} } \\
\text { (8) } \quad= & {\left[\exp \left\{-\int_{n}^{n+\frac{1}{2}} A(s) \mathrm{d} s\right\}+\int_{n}^{n+\frac{1}{2}} B(u) \exp \left\{-\int_{u}^{n+\frac{1}{2}} A(s) \mathrm{d} s\right\} \mathrm{d} u\right] a_{n} . }
\end{aligned}
$$

Multiplying both sides of (8) by $\exp \left\{\int_{n+1}^{n+\frac{1}{2}} A(s) \mathrm{d} s\right\}$ yields

$$
\begin{gathered}
{\left[I+\int_{n+1}^{n+\frac{1}{2}} B(u) \exp \left\{-\int_{u}^{n+1} A(s) \mathrm{d} s\right\} \mathrm{d} u\right] a_{n+1}} \\
=\left[\exp \left\{-\int_{n}^{n+1} A(s) \mathrm{d} s\right\}+\int_{n}^{n+\frac{1}{2}} B(u) \exp \left\{-\int_{u}^{n+1} A(s) \mathrm{d} s\right\} \mathrm{d} u\right] a_{n},
\end{gathered}
$$

which is equivalent to

$$
\begin{gathered}
{\left[I-\int_{n+\frac{1}{2}}^{n+1} B(u) \exp \left\{-\int_{u}^{n+1} A(s) \mathrm{d} s\right\} \mathrm{d} u\right] a_{n+1}} \\
=\left[\exp \left\{-\int_{n}^{n+1} A(s) \mathrm{d} s\right\}+\int_{n}^{n+\frac{1}{2}} B(u) \exp \left\{-\int_{u}^{n+1} A(s) \mathrm{d} s\right\} \mathrm{d} u\right] a_{n} .
\end{gathered}
$$

Since the matrix $C_{n}$ is non-singular, we see that

$$
\begin{aligned}
a_{n+1}= & {\left[I-\int_{n+\frac{1}{2}}^{n+1} B(u) \exp \left\{-\int_{u}^{n+1} A(s) \mathrm{d} s\right\} \mathrm{d} u\right]^{-1} } \\
& \times\left[\exp \left\{-\int_{n}^{n+1} A(s) \mathrm{d} s\right\}+\int_{n}^{n+\frac{1}{2}} B(u) \exp \left\{-\int_{u}^{n+1} A(s) \mathrm{d} s\right\} \mathrm{d} u\right] a_{n},
\end{aligned}
$$

or in the simple form

$$
a_{n+1}=C_{n}^{-1} D_{n} a_{n}, \quad n=0,1, \ldots
$$


where

$$
C_{n}=I-\int_{n+\frac{1}{2}}^{n+1} B(u) F(n+1, u) \mathrm{d} u, \quad F(u, v)=\exp \left\{-\int_{v}^{u} A(s) \mathrm{d} s\right\}
$$

and

$$
D_{n}=F(n+1, n)+\int_{n}^{n+\frac{1}{2}} B(u) F(n+1, u) \mathrm{d} u,
$$

which proves the Theorem.

Remark 2.1. Taking $A(t)=-A$ and $B(t)=B$ in Theorem 2.1 above, we get Theorem 1 in [9]. In this case, Eq. (3) takes the form

$$
x(t)=\left[\mathrm{e}^{A(t-n)}+\int_{n}^{t} B \mathrm{e}^{A(t-u)} \mathrm{d} u\right] a_{n},
$$

and

$$
x(t)=\left[\mathrm{e}^{A}(t-n)-\left(I-\mathrm{e}^{A(t-n)}\right) A^{-1} B\right] a_{n} .
$$

Therefore

$$
x(t)=M(t-n) a_{n}, \quad a_{n}=x(n),
$$

where

$$
M(t)=\mathrm{e}^{A t}+\left(\mathrm{e}^{A t}-I\right) A^{-1} B
$$

and we obtain the result in [9].

Remark 2.2. If $A$ and $B$ are constant matrices and $\left[t+\frac{1}{2}\right]$ is replaced by $[t]$, we get Theorem 1.1 in [2].

Theorem 2.2. The solution $x(t)$ of Eq. (2) has a unique backward continuation on $(-\infty, 0)$ given by $(3)$ and

$$
a_{n-1}=W_{n}^{-1} Z_{n} a_{n}, \quad n=0,-1,-2, \ldots
$$

where the matrices $W_{n}$ and $Z_{n}$ are given by

$$
\left\{\begin{array}{l}
W_{n}=I+\int_{n-1}^{n-\frac{1}{2}} B(u) \exp \left\{-\int_{u}^{n-1} A(s) \mathrm{d} s\right\} \mathrm{d} u \\
Z_{n}=\exp \left\{-\int_{n}^{n-1} A(s) \mathrm{d} s\right\}+\int_{n}^{n-\frac{1}{2}} B(u) \exp \left\{-\int_{u}^{n-1} A(s) \mathrm{d} s\right\} \mathrm{d} u
\end{array}\right.
$$

provided the matrix $W_{n}$ is non-singular. 
Pr o of. As in the proof of Theorem 2.1, for $t \in\left[n-\frac{1}{2}, n+\frac{1}{2}\right)$ we have Eq. (3) and as $t \rightarrow n-\frac{1}{2}$, we get

(10) $x\left(n-\frac{1}{2}\right)=\left[\exp \left\{-\int_{n}^{n-\frac{1}{2}} A(s) \mathrm{d} s\right\}+\int_{n}^{n-\frac{1}{2}} B(u) \exp \left\{-\int_{u}^{n-\frac{1}{2}} A(s) \mathrm{d} s\right\} \mathrm{d} u\right] a_{n}$.

Also, for $t \in\left[n-\frac{3}{2}, n-\frac{1}{2}\right)$, integrating Eq. (2) from $n-1$ to $t$ and letting $t \rightarrow n-\frac{1}{2}$ we obtain

$$
x\left(n-\frac{1}{2}\right)=\left[\exp \left\{-\int_{n-1}^{n-\frac{1}{2}} A(s) \mathrm{d} s\right\}+\int_{n-1}^{n-\frac{1}{2}} B(u) \exp \left\{-\int_{u}^{n-\frac{1}{2}} A(s) \mathrm{d} s\right\} \mathrm{d} u\right] a_{n-1} .
$$

From Equations (10) and (11) we find

$$
\begin{aligned}
& {\left[\exp \left\{-\int_{n-1}^{n-\frac{1}{2}} A(s) \mathrm{d} s\right\}+\int_{n-1}^{n-\frac{1}{2}} B(u) \exp \left\{-\int_{u}^{n-\frac{1}{2}} A(s) \mathrm{d} s\right\} \mathrm{d} u\right] a_{n-1}} \\
& =\left[\exp \left\{-\int_{n}^{n-\frac{1}{2}} A(s) \mathrm{d} s\right\}+\int_{n}^{n-\frac{1}{2}} B(u) \exp \left\{-\int_{u}^{n-\frac{1}{2}} A(s) \mathrm{d} s\right\} \mathrm{d} u\right] a_{n},
\end{aligned}
$$

which is equivalent to

$$
\begin{gathered}
{\left[I+\int_{n-1}^{n-\frac{1}{2}} B(u) \exp \left\{-\int_{u}^{n-1} A(s) \mathrm{d} s\right\} \mathrm{d} u\right] a_{n-1}} \\
=\left[\exp \left\{-\int_{n}^{n-1} A(s) \mathrm{d} s\right\}+\int_{n}^{n-\frac{1}{2}} B(u) \exp \left\{-\int_{u}^{n-1} A(s) \mathrm{d} s\right\} \mathrm{d} u\right] a_{n} .
\end{gathered}
$$

Hence we have

$$
a_{n-1}=W_{n}^{-1} Z_{n} a_{n}, \quad n=0,-1, \ldots
$$

where $W_{n}$ and $Z_{n}$ are defined by (9). The proof is completed.

Theorem 2.3. Assume that

$$
|A(t)| \geqslant \delta \text { for some } \delta>0 \text { and }|B(t)| \leqslant \gamma \text { where } 0<\gamma<\delta .
$$

Then the trivial solution of Eq. (2) is globally asymptotically stable.

Pro of. Let $x(t)$ be a solution of Eq. (2). From Eq. (3) we have

$$
|x(t)|=|Z(t)|\left|a_{n}\right|,
$$

where

$$
Z(t)=\exp \left\{-\int_{n}^{t} A(s) \mathrm{d} s\right\}+\int_{n}^{t} B(u) \exp \left\{-\int_{u}^{t} A(s) \mathrm{d} s\right\} \mathrm{d} u
$$


It follows from Condition (i) that

$$
|Z(t)| \leqslant \mathrm{e}^{-\delta(t-n)}+\gamma \int_{n}^{t} \mathrm{e}^{-\delta(t-u)} \mathrm{d} u \leqslant 1+\frac{\gamma}{\delta}\left[1-\mathrm{e}^{-\delta(t-n)}\right] .
$$

Then we get

$$
|Z(t)| \leqslant 1+\frac{\gamma}{\delta}
$$

Now Eq. (4) implies

$$
a_{n+1}=T_{n} a_{n}, \quad n=0,1, \ldots
$$

where $T_{n}=C_{n}^{-1} D_{n}$. Hence we obtain

$$
a_{n}=T_{n-1} T_{n-2} \ldots T_{0} a_{0}, \quad a_{0}=x(0), \quad n=0,1, \ldots
$$

Thus

$$
\left|a_{n}\right|=\left|T_{n-1}\right|\left|T_{n-2}\right| \ldots\left|T_{0}\right|\left|a_{0}\right| .
$$

Now, Condition (i) gives

$$
\left|D_{n}\right| \leqslant \mathrm{e}^{-\delta}+\gamma \int_{n}^{n+\frac{1}{2}} \mathrm{e}^{-\delta(n+1-u)} \mathrm{d} u, \quad n=0,1, \ldots
$$

and so

$$
\left|D_{n}\right| \leqslant \mathrm{e}^{-\delta}+\frac{\gamma}{\delta}\left[\mathrm{e}^{-\delta / 2}-\mathrm{e}^{-\delta}\right] .
$$

Let $H=\int_{n+\frac{1}{2}}^{n+1} B(u) \exp \left\{-\int_{u}^{n+1} A(s) \mathrm{d} s\right\} \mathrm{d} u$. Then by using Condition (i), we have

$$
|H| \leqslant \gamma \int_{n+\frac{1}{2}}^{n+1} \mathrm{e}^{-\delta(n+1-u)} \mathrm{d} u \leqslant \frac{\gamma}{\delta}\left[1-\mathrm{e}^{-\delta / 2}\right] .
$$

Therefore, from the fact that $|A|-|B| \leqslant|| A|-| B|| \leqslant|A-B|$ for the $p \times q$ matrices $A$ and $B$, we find

$$
\left|C_{n}\right|=|I-H| \geqslant|I|-|H| \geqslant 1-\frac{\gamma}{\delta}\left[1-\mathrm{e}^{-\delta / 2}\right] .
$$

Thus Inequalities (15) and (16) give

$$
\left|T_{n}\right|=\left|C_{n}^{-1}\right|\left|D_{n}\right|=\left|C_{n}\right|^{-1}\left|D_{n}\right| \leqslant \frac{\mathrm{e}^{-\delta}+\frac{\gamma}{\delta}\left[\mathrm{e}^{-\delta / 2}-\mathrm{e}^{-\delta}\right]}{1-\frac{\gamma}{\delta}\left[1-\mathrm{e}^{-\delta / 2}\right]} .
$$


Now we obtain $\frac{\gamma}{\delta}<1$ from (i). Consequently

$$
\frac{\gamma}{\delta}\left[1-\mathrm{e}^{-\delta}\right]<1-\mathrm{e}^{-\delta}
$$

Thus

$$
\frac{\gamma}{\delta}\left[1-\mathrm{e}^{-\delta}+\mathrm{e}^{-\delta / 2}-\mathrm{e}^{-\delta / 2}\right]<1-\mathrm{e}^{-\delta} .
$$

Hence we have

$$
\frac{\gamma}{\delta}\left[1-\mathrm{e}^{-\delta / 2}\right]+\frac{\gamma}{\delta}\left[\mathrm{e}^{-\delta / 2}-\mathrm{e}^{-\delta}\right]<1-\mathrm{e}^{-\delta}
$$

or

$$
\mathrm{e}^{-\delta}+\frac{\gamma}{\delta}\left[\mathrm{e}^{-\delta / 2}-\mathrm{e}^{-\delta}\right]<1-\frac{\gamma}{\delta}\left[1-\mathrm{e}^{-\delta / 2}\right] .
$$

Therefore we obtain

$$
\frac{\mathrm{e}^{-\delta}+\frac{\gamma}{\delta}\left[\mathrm{e}^{-\delta / 2}-\mathrm{e}^{-\delta}\right]}{1-\frac{\gamma}{\delta}\left[1-\mathrm{e}^{-\delta / 2}\right]}<1 .
$$

Now from (17) we get

$$
\left|T_{n}\right| \leqslant \lambda<1, \quad n=0,1, \ldots
$$

where

$$
\lambda=\frac{\mathrm{e}^{-\delta}+\frac{\gamma}{\delta}\left[\mathrm{e}^{-\frac{\delta}{2}}-\mathrm{e}^{-\delta}\right]}{1-\frac{\gamma}{\delta}\left[1-\mathrm{e}^{-\frac{\delta}{2}}\right]} .
$$

Thus Equations (14) and (18) give

$$
\left|a_{n}\right| \leqslant \lambda^{n}\left|a_{0}\right|
$$

which implies

$$
\lim _{n \rightarrow \infty} a_{n}=0 .
$$

Thus it follows from (12), (13), and (19) that

$$
\lim _{t \rightarrow \infty} x(t)=0
$$

and the proof is completed.

456 
Example. Consider the differential equation

$$
x^{\prime}(t)=A(t) x(t)+B(t) x\left(\left[t+\frac{1}{2}\right]\right), \quad t \geqslant 0
$$

where $x(t) \in \mathbb{R}^{2}$ and $A(t)$ and $B(t)$ are $2 \times 2$ matrices given by

$$
A(t)=\left[\begin{array}{cc}
\sin t-1 & \cos t \\
\frac{1-\mathrm{e}^{-t^{2}}}{\mathrm{e}} & \frac{-\lambda}{1+\mathrm{e}^{-t}}
\end{array}\right]
$$

and

$$
B(t)=\left[\begin{array}{cc}
-\mathrm{e}^{-\beta} \sin t & -\mathrm{e}^{-\beta} \cos t \\
\frac{\mathrm{e}^{-t^{2}}-2}{\mathrm{e}^{\beta+1}} & \frac{\lambda \mathrm{e}^{-\beta}}{1+\mathrm{e}^{-t}}
\end{array}\right]
$$

for some constants $\lambda>2$ and $\beta>0$. By Theorem 2.3, the trivial solution of Eq. (20) is globally asymptotically stable. In fact, the vector $x(t)=\left(\mathrm{e}^{-t}, \mathrm{e}^{-(t+1)}\right)$ is a solution of Eq. (20).

II.

In this section we study Eq. (1).

Theorem 2.4. Assume that the matrix $C_{n}$ defined as in Theorem 2.1 is also non-singular. Then Eq. (1) has a unique solution on $[0, \infty)$ given by

$$
\begin{aligned}
x(t)= & {\left[\exp \left\{-\int_{n}^{t} A(s) \mathrm{d} s\right\}+\int_{n}^{t} B(u) \exp \left\{-\int_{u}^{t} A(s) \mathrm{d} s\right\} \mathrm{d} u\right] a_{n} } \\
& +\int_{n}^{t} \exp \left\{-\int_{u}^{t} A(s) \mathrm{d} s\right\} f\left(u, x(u), a_{n}\right) \mathrm{d} u,
\end{aligned}
$$

where $a_{n}$ is a solution of the difference equation

$$
\begin{gathered}
a_{n+1}=T_{n} a_{n}+W_{n}, \quad n=0,1, \ldots, \\
T_{n}=C_{n}^{-1} D_{n}, \quad W_{n}=C_{n}^{-1}\left(E_{n}+F_{n}\right),
\end{gathered}
$$

the matrices $C_{n}$ and $D_{n}$ are defined as in Theorem 2.1 and the matrices $E_{n}$ and $F_{n}$ are defined by

$$
\left\{\begin{array}{l}
E_{n}=\int_{n+\frac{1}{2}}^{n+1} \exp \left\{-\int_{u}^{n+1} A(s) \mathrm{d} s\right\} f\left(u, x(u), a_{n+1}\right) \mathrm{d} u \\
F_{n}=\int_{n}^{n+\frac{1}{2}} \exp \left\{-\int_{u}^{n+1} A(s) \mathrm{d} s\right\} f\left(u, x(u), a_{n}\right) \mathrm{d} u .
\end{array}\right.
$$

Proof. The proof is similar to that of Theorem 2.1 and therefore it is omitted. 
Theorem 2.5. Assume that Condition (i) in Theorem 2.3 holds. Let $f(t, x, y), t \in$ $\mathbb{R}, x, y \in \mathbb{R}^{r}$ satisfy

$$
\left|f\left(t, x_{1}, y_{1}\right)-f\left(t, x_{2}, y_{2}\right)\right| \leqslant N(t)\left|x_{1}-x_{2}\right|+K(t)\left|y_{1}-y_{2}\right|
$$

where $N(t)$ and $K(t)$ are non-negative scalar functions defined on $\left(t_{0}, \infty\right)$ such that

$$
\int_{t_{0}}^{\infty} N(s) \mathrm{d} s=\alpha<\infty, \quad \text { and } \quad \int_{t_{0}}^{\infty} K(s) \mathrm{d} s=\beta<\infty, \quad \alpha, \beta \in \mathbb{R}^{+} .
$$

Then the trivial solution of Eq. (1) is globally asymptotically stable.

Pr o of. From Eq. (21) we find

$$
|x(t)| \leqslant|z(t)|\left|a_{n}\right|+\left|\int_{n}^{t} \exp \left\{-\int_{u}^{t} A(s) \mathrm{d} s\right\} f\left(u, x(u), a_{n}\right) \mathrm{d} u\right|,
$$

where $z(t)$ is defined as in Theorem 2.3. Now, it follows from Conditions (i) and (ii) that

$$
\begin{aligned}
& \left|\int_{n}^{t} \exp \left\{-\int_{u}^{t} A(s) \mathrm{d} s\right\} f\left(u, x(u), a_{n}\right) \mathrm{d} u\right| \\
& \leqslant \int_{n}^{t} \exp \left\{-\int_{u}^{t} A(s) \mathrm{d} s\right\}\left[N(u)|x(u)|+K(u)\left|a_{n}\right|\right] \mathrm{d} u \\
& \leqslant \int_{n}^{t} N(u)|x(u)| \mathrm{d} u+\int_{n}^{t} K(u)\left|a_{n}\right| \mathrm{d} u .
\end{aligned}
$$

By Condition (iii) we obtain

$$
\left|\int_{n}^{t} \exp \left\{-\int_{u}^{t} A(s) \mathrm{d} s\right\} f\left(u, x(u), a_{n}\right) \mathrm{d} u\right| \leqslant \int_{n}^{t} N(u)|x(u)| \mathrm{d} u+\beta\left|a_{n}\right| .
$$

By substituting from (25) in (24), we get

$$
|x(t)| \leqslant(|z(t)|+\beta)\left|a_{n}\right|+\int_{n}^{t} N(u)|x(u)| \mathrm{d} u .
$$

Eq. (22) implies

$$
a_{n}=C_{n-1}^{-1} D_{n-1} a_{n-1}+W_{n-1}, \quad n=1,2, \ldots
$$

Therefore from Theorem 2.1 in $[6$, p. 13] we have

$$
a_{n}=T_{n-1} T_{n-2} \ldots T_{0} a_{0}+\sum_{r=0}^{n-1} T_{n-1} T_{n-2} \ldots T_{r} W_{r} .
$$

458 
Thus

$$
\left|a_{n}\right| \leqslant\left|T_{n-1}\right|\left|T_{n-2}\right| \ldots\left|T_{0}\right|\left|a_{0}\right|+\sum_{r=0}^{n-1}\left|T_{n-1}\right|\left|T_{n-2}\right| \ldots\left|T_{r}\right|\left|W_{r}\right| .
$$

Also as in Theorem 2.3, from (18) we find

$$
\left|a_{n}\right| \leqslant \lambda^{n}\left|a_{0}\right|+\sum_{r=0}^{n-1} \lambda^{n-1-r}\left|W_{r}\right|
$$

Now

$$
\begin{aligned}
\left|W_{n}\right|=\left|C_{n}^{-1}\right| \mid & \int_{n+\frac{1}{2}}^{n+1} \exp \left\{-\int_{u}^{n+1} A(s) \mathrm{d} s\right\} f\left(u, x(u), a_{n+1}\right) \mathrm{d} u \\
& +\int_{n}^{n+\frac{1}{2}} \exp \left\{-\int_{u}^{n+1} A(s) \mathrm{d} s\right\} f\left(u, x(u), a_{n+1}\right) \mathrm{d} u \mid .
\end{aligned}
$$

Assume that

$$
\overline{a_{n}}=\max _{n \leqslant t \leqslant n+1}\{|x(t)|\} .
$$

Then by Condition (i) we have

$$
\left|W_{n}\right| \leqslant\left|C_{n}^{-1}\right| \int_{n}^{n+1} 2 \mathrm{e}^{-\delta(n+1-u)}[N(u)+K(u)]\left|\overline{a_{n}}\right| \mathrm{d} u .
$$

So, from Inequality (16) and Condition (iii), we see that

$$
\left|W_{n}\right| \leqslant \frac{2(\alpha+\beta)}{1-\frac{\gamma}{\delta}\left(1-\mathrm{e}^{-\delta}\right)}\left|\overline{a_{n}}\right|=L\left|\overline{a_{n}}\right|,
$$

where $L=\frac{2 \delta(\alpha+\beta)}{\delta-\gamma\left(1-\mathrm{e}^{-\delta}\right)}$. Then it follows from (27) and (28) that

$$
\left|\overline{a_{n}}\right| \leqslant \lambda^{n}\left|a_{0}\right|+\sum_{r=0}^{n-1} L \lambda^{n-1-r}\left|\overline{a_{r}}\right|
$$

Applying the discrete Gronwall's inequality [6, p. 21] to Eq. (20), we obtain

$$
\left|\overline{a_{n}}\right| \leqslant \lambda^{n}\left|a_{0}\right| \exp \left\{L \sum_{r=0}^{n-1} \lambda^{n-1-r}\right\}=\lambda^{n}\left|a_{0}\right| \mathrm{e}^{L\left(\lambda^{n-1}+\lambda^{n-2}+\ldots+1\right)} .
$$

Therefore, we have

$$
\left|a_{n}\right| \leqslant \lambda^{n}\left|a_{0}\right| \mathrm{e}^{L \lambda^{n} /(1-\lambda)} .
$$


In view of (26) and (30), we obtain

$$
|x(t)| \leqslant(|z(t)|+\beta) \lambda^{n}\left|a_{0}\right| \mathrm{e}^{L \lambda^{n} /(1-\lambda)}+\int_{n}^{t} N(u)|x(u)| \mathrm{d} u .
$$

Thus Inequality (13) implies

$$
|x(t)| \leqslant\left(1+\frac{\gamma}{\delta}+\beta\right) \lambda^{n}\left|a_{0}\right| \mathrm{e}^{L \lambda^{n} /(1-\lambda)}+\int_{n}^{t} N(u)|x(u)| \mathrm{d} u .
$$

Again applying the continuous Gronwall's inequality we obtain

$$
|x(t)| \leqslant\left(1+\frac{\gamma}{\delta}+\beta\right) \lambda^{n}\left|a_{0}\right| \mathrm{e}^{L \lambda^{n} /(1-\lambda)} \exp \left\{\int_{n}^{t} N(u) \mathrm{d} u\right\},
$$

which by Condition (ii) gives

$$
|x(t)| \leqslant\left(1+\frac{\gamma}{\delta}+\beta\right) \lambda^{n}\left|a_{0}\right| \mathrm{e}^{\alpha} \mathrm{e}^{L \lambda^{n} /(1-\lambda)} .
$$

Since $\frac{L}{1-\lambda}>0$, we obtain

$$
|x(t)| \leqslant\left(1+\frac{\gamma}{\delta}+\beta\right)\left|a_{0}\right| \mathrm{e}^{\alpha} \lambda^{t} \mathrm{e}^{L \lambda^{t} /(1-\lambda)} .
$$

Thus

$$
\lim _{t \rightarrow \infty} x(t)=0 .
$$

The proof is completed.

\section{References}

[1] A. R. Aftabizadeh and J. Wiener: Oscillatory and periodic solutions of an equation alternately of retarded and advanced type. Appl. Anal. 23 (1986), 219-231.

[2] A. R. Aftabizadeh and J. Wiener: Oscillatory and periodic solutions for systems of two first order linear differential equations with piecewise constant argument. Appl. Anal. 26 (1988), 327-333.

[3] S. Busenderg and K. L. Cooke: Models of vertically transmitted diseases with sequential-continuous dynamics. In: Nonlinear Phenomena in Mathematical Science (V. Lakshmikantham, ed.). Academic Press, New York, 1982, pp. 179-187.

[4] K. L. Cooke and J. Wiener: Retarded differential equation with piecewise constant delays. J. Math. Anal. Appl. 99 (1984), 265-297.

[5] K. L. Cooke and J. Wiener: An equation alternately of retarded and advanced type. Proc. Amer. Math. Soc. 99 (1987), 726-732.

[6] K. Miller: Linear Difference Equations. Benjamin, New York, 1968.

[7] S.M. Shah and J. Wiener: Advanced differential equations with piecewise constant argument deviations. Internat. J. Math. Sci. 6 (1983), 671-703. 
[8] J. Wiener and A.R. Aftabizadeh: Differential equations alternately of retarded and advanced type. J. Math. Anal. Appl. 129 (1988), 243-255.

[9] J. Wiener and K. L. Cooke: Oscillations in systems of differential equations with piecewise constant argument. J. Math. Anal. Appl. 137 (1989), 221-239.

Authors' addresses: S. R. Grace, Department of Engineering Mathematics, Faculty of Engineering, Cairo University, Orman, Giza 12211, Egypt, e-mail: srgrace@alpha1eng.cairo.eun.eg; M.R.S. Kulenovi 'cand H. El-Metwally, Department of Mathematics, University of Rhode Island, Kingston, RI 02881, USA, e-mail: kulenm@math. uri.edu. 